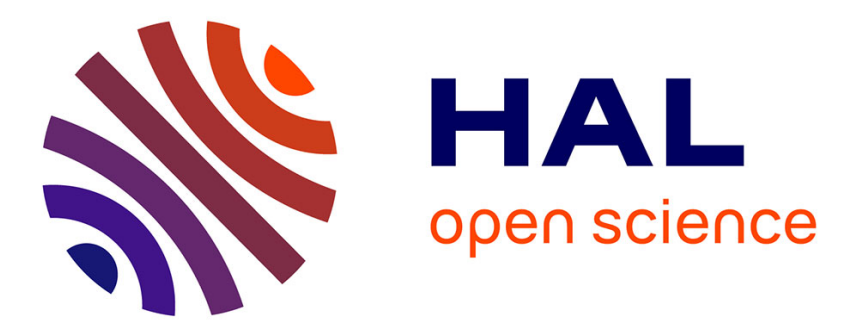

\title{
CAN WE PREDICT SELF-REPORTED CUSTOMER SATISFACTION FROM INTERACTIONS?
}

\author{
Jeremy Auguste, Delphine Charlet, Geraldine Damnati, Frédéric Béchet, \\ Benoit Favre
}

\section{- To cite this version:}

Jeremy Auguste, Delphine Charlet, Geraldine Damnati, Frédéric Béchet, Benoit Favre. CAN WE PREDICT SELF-REPORTED CUSTOMER SATISFACTION FROM INTERACTIONS?. 2019 IEEE International Conference on Acoustics, Speech and Signal Processing (ICASSP), May 2019, Brighton, United Kingdom. 10.1109/ICASSP.2019.8683896 . hal-02439687

\section{HAL Id: hal-02439687 https://hal.science/hal-02439687}

Submitted on 14 Jan 2020

HAL is a multi-disciplinary open access archive for the deposit and dissemination of scientific research documents, whether they are published or not. The documents may come from teaching and research institutions in France or abroad, or from public or private research centers.
L'archive ouverte pluridisciplinaire HAL, est destinée au dépôt et à la diffusion de documents scientifiques de niveau recherche, publiés ou non, émanant des établissements d'enseignement et de recherche français ou étrangers, des laboratoires publics ou privés. 


\title{
CAN WE PREDICT SELF-REPORTED CUSTOMER SATISFACTION FROM INTERACTIONS?
}

\author{
Jeremy Auguste $^{1} \quad$ Delphine Charlet $^{2} \quad$ Geraldine Damnati $^{2} \quad$ Frederic Bechet $^{1} \quad$ Benoit Favre $^{1}$ \\ (1) Aix Marseille Univ, Université de Toulon, CNRS, LIS, Marseille, France \\ (2) Orange Labs, Lannion, France \\ \{jeremy.auguste, frederic.bechet, benoit.favre\}@lis-lab.fr \\ \{delphine.charlet, geraldine.damnati\}@orange.com
}

\begin{abstract}
In the context of contact centers, customers' satisfaction after a conversation with an agent is a critical issue which has to be collected in order to detect problems and improve quality of service. Automatically predicting customer satisfaction directly from system logs, without any survey or manual annotation is a challenging task of a great interest for the field of human-human conversation understanding and for improving contact center quality of service. Unlike previous studies that have focused on questions directly related to the content of a conversation, we look at a more general opinion about a service which is called the "Net Promoter Score" (NPS) where customers are considered either as promoters, detractors or neutral. On a very large corpus of chat-conversations with customer satisfaction surveys, we explore several classification scheme in order to achieve this prediction task, only using conversation logs.
\end{abstract}

Index Terms - Human-Human conversation mining, Net Promoter Score, Opinion Analysis, CNN models, Attentionbased RNN models

\section{INTRODUCTION}

Contact centers from large companies like telephone or internet companies are a unique opportunity to study real humanhuman conversations between customers and operators.

Predicting automatically self-reported feedback to satisfaction survey after a conversation without asking customers is a very challenging scientific task of great practical application. This task was studied by previous studies in the context of human-computer interaction and call-centers satisfaction surveys $[1,2]$. These studies targeted questions directly related to a given conversation with an operator or a machine. In this study we look at a more general opinion about a service which goes beyond a single conversation, called the Net Promoter Score.

One of the most observed indicator in Customer Relationship Management is the Net Promoter Score (NPS). Beyond satisfaction, customers are asked if they would recommend the company. In most cases, this recommendation has to be given on a scale ranging from 0 to 10 . Customers who answer from 9 to 10 are considered as promoters, those who answer from 0 to 6 are considered as detractors and the others are considered as neutral or passive. The NPS is the difference between promoters and detractors. Companies expect this difference to be positive, and to be as high as possible.

In this paper we are interested in studying to what extent the NPS could be predicted directly from conversation logs. On a large corpus of technical and commercial conversation chats, we compare several classification methods and answer the two following questions thanks to contrastive experiments and detailed performance analyses: Which metrics should be optimized in order to estimate NPS from a corpus of conversation logs? Can we estimate accurately the promoters and detractors distribution using only system logs?

\section{RELATED WORK}

In order to train classification models to evaluate customers satisfaction from system logs two kinds of supervision can be used: direct supervision thanks to self-reported customer satisfaction through surveys filled by customers after a conversation $[1,2]$ and indirect supervision produced by experts from conversation logs $[3,4,5]$. For highly subjective measures such as NPS, only direct supervision through self-reported satisfaction can be used. As discussed in [1], this use of selfreported satisfaction raises 3 issues:

- only a small percentage of customers fill surveys leading to a lack of annotated data with possible biases;

- human rating is often asked with a 5 or a 10 levels scale, should we consider each scale as a label, use regression instead of classification or group together scales to obtain labels?

- the last issue is about the feasibility of the task itself for subjective questions related to a global satisfaction which goes beyond the current conversation, such as 
the NPS: do conversations contain enough objective evidence about these subjective opinions?

The first issue is not problematic in our case because we are using chat data: customers are more likely to click on a few boxes on a web interface after a chat than answering questions orally. Indeed a study on our corpus shows that $30 \%$ of the chat conversations have a survey, therefore we have no lack of data considering the size of the corpus we are processing in this study.

For the second issue, [1] addresses the problem of customer satisfaction prediction from call-center telephone conversations with observations from the field of emotion annotation, stating that human ratings of emotion or opinion do not follow an absolute scale. Hence, they adopted the ordinal ranking paradigm in order to address the categorical scale issue. In this study we overcome this issue by using a mapping from rating scores to categories (detractor,passive,promoter) provided by the Customer Relationship Management field.

The last issue is one of the main motivation of this study: can we retrieve directly from conversation logs such subjective opinions as NPS? By comparing results obtained by several state-of-the-art classifiers trained on very large quantities of data we will try to answer this question.

\section{CONVERSATION CHAT DATA AND SURVEYS}

\subsection{Chat data description}

Chat conversations are extracted from Orange customer services contact center logs. The corpus covers a wide variety of topics, ranging from technical issues (eg. solving a connexion problem) to commercial inquiries (eg. purchasing a new offer). They can cover several applicative domains (mobile, internet, tv). In [6], we compared these chat conversations with spoken telephone conversations from equivalent contact centers. Even though chat conversation and telephone conversation present some obvious differences, they contain similar language register (spontaneous but formal interaction) and similar interaction properties.

From a Language Understanding point of view, processing spoken conversations or chat conversations both imply noisy input phenomena (ASR errors and disfluencies for the former and orthographic deviations for the latter). We have evaluated the ratio of orthographic deviations, that can be assimilated to a Word Error Rate for chat conversations. This WER is $4.3 \%$ on the overall with an unbalanced distribution of errors: about $10.1 \%$ for Customers and $1.6 \%$ for Agents.

\subsection{Customer surveys}

At the end of a conversation, customers have the option to fill in a survey among which can be found several questions regarding various specific dimensions (e.g. satisfaction on the quality of explanations, advises, support, solutions,...) as well as a more general question: "Considering your contact with our company, how likely would you be to recommend us to your friends or family?". This question reflects a general appreciation for which customers can express a broader feeling than the conversation per se. It is a Key Performance Indicator that is closely monitored for Customer Relationship Management (CRM). For this latter question, the customer is required to provide an answer along a scale ranging from 0 to 10. Following CRM conventions, these appreciations can be grouped into 3 categories: detractors (from 0 to 6), passives (7 or 8 ) and promoters ( 9 or 10). Data have been collected over a one month period, and we have selected the subset of conversations for which the customer has provided an answer to all questions. The training, development and test corpora are respectively constituted of $47,685,15,899$ and 15,892 conversations. The training corpus is composed of 140,000 different tokens. As mentioned in the previous section, the originality of this study is that the annotation supervision is directly provided by the protagonist. There are as many annotators as conversations. The large amount of training and test data with this self-reported direct supervision (almost 80k conversations) is also relatively unusual for this type of study.

\section{PREDICTING SELF-REPORTED SATISFACTION}

In this study we consider the problem of predicting if a customer is likely to recommend the company as a supervised classification problem where a set of classifiers using different textual representations are trained to predict a label for each customer interaction, among the set $\{$ detractor, passive or promoter $\}$.

We compare several classification models trained following 3 different schemes:

- 3-label classification scheme: in this scheme we use a single classifier where all the three labels to predict have the same weight;

- $2 \times 2$-label classification scheme: we consider here two binary (yes/no) classifiers, the first one in charge of predicting the detractor label, the second one predicting the promoter label; the passive label is predicted when both binary classifiers return no;

- 2-label multitask classification scheme: this is a variation of the previous scheme where only one classifier is used in a multitask process; we consider the prediction of the detractor and promoter labels as two tasks jointly performed, when both prediction are negative, the label given is the passive label.

We define these schemes since the 3 labels to predict are not equivalent from our applicative point of view: the NPS being the difference between the proportions of promoters and detractors, a confusion between these two labels or with the passive label do not have the same applicative impact. 
We have implemented these schemes with three different state-of-the-art classification methods: Support Vector Machines (SVM) with a linear kernel and a bag-of-word representation; Convolutional Neural Networks (CNN) with contiguous word blocks and Recurrent Neural Networks (RNN) with word and turn sequences.

For SVM the only information about the structure of the conversations that is kept is the speaker of each word. To do that, each word is concatenated with its speaker. For all experiments involving deep neural networks, we used embeddings of dimension 100, with no-pretraining. For the CNN model, we use the same architecture as described in [7]. We build our model with filters of size 3,4 and 5 and 100 filters for each size. The RNN model is based on a Long Short Term Memory (LSTM) model with an attention mechanism $[8,9]$ which allows the model to focus on the important words in each conversation with respect to the targeted task. The hidden layers in the LSTM have a size of 128.

The 2-label multitask classification scheme is implemented only with the CNN and RNN classifiers. In this case, the 2 detectors are not trained independently, but trained jointly in order to share all the layers except the decision ones devoted to the specific classification.

\section{EXPERIMENTS}

In order to compare the different models and classification schemes, we use 4 metrics:

- accuracy: classification accuracy simply measures the percentage of correct decision;

- F1: this is the F-measure, estimated for each label $l$ as the harmonic mean of precision $P$ and recall $R$ for $l$ such as $F 1(l)=\frac{2 \times P \times R}{P+R}$

- macroF1: this is the non-weighted average of the F1 measure over our 3 labels;

- SER (Serious Error Rate): we consider a confusion between labels detractor and promoter being more serious than one with the passive label, since the impact on the NPS will be greater; therefore we define the SER as the percentage of confusion between these two extreme classes.

\subsection{Comparing classification methods and schemes}

Table 1 reports results obtained by using different types of models with different classification schemes. It can be noted that all models achieve better results than simply taking the majority class by at least 10 points on the accuracy and 26 points on the macroF1. However, the highest accuracy and macroF1 are only of $57.5 \%$ and $53.8 \%$ respectively. These results are significantly lower than previous reported results on customer satisfaction prediction such as [3], however we have to keep in mind that we are dealing here with self-reported satisfaction not only about a given call, but about a general opinion on the service and the company, making the task much harder.

The 3-label and 2-label schemes clearly show different behaviors: the 3-label obtains the best accuracy although the 2label favors the macroF1 and SER metrics. The 3-label classification scheme obtains very poor results for predicting the passive label. This can be explained by the difficulty of modelling directly this passive class that is much more diverse than the other classes which should contain some explicit content for justifying the positive or negative opinions.

Comparatively, all the models that use a 2-label with rejection classification scheme greatly improve the prediction of the passive class with $\mathrm{F} 1$-scores ranging from $36 \%(\mathrm{CNN})$ to $40 \%$ (SVM, RNN). And even if the F1-scores of the other two labels are slightly lower by 3 to 6 points, the improvements on the passive class allows us to improve the macroF1 score by at least 5 points on all models. The SER is also greatly reduced by the 2-label+rejection classification scheme models.

This is an expected behavior coming from the fact that in the 3-label models any bad prediction of a promoter/detractorlabeled conversation would result in a serious error since they barely ever use the passive label, whereas the 2-label models can predict that a sample is labeled with neither promoter nor detractor. The main drawback of the 2-label models is that they get $2(\mathrm{CNN})$ to 4 (SVM, RNN) fewer points in accuracy than the 3-label models.

Among the 2-label models, it is interesting to note that multitask and independent models achieve very similar results. However, the independent models require to train two distinct models, thus it takes twice as long to do the training.

As a first conclusion we can argue that the classification scheme to choose depends on the metrics to optimize. In our case, since the NPS score depends only on the estimation of the correct proportion of detractor and promoter customers, the macro F1 and the SER are more important than the accuracy, therefore we choose the 2-label scheme.

All classification methods for the 2-label scheme achieve rather similar performance, although $\mathrm{CNN}$ is slightly better in terms of macro F1.

\subsection{Contrastive experiments}

In a first experiment, we want to know which speaker's turns are the most important to the task. It is expected that the client's turn input the most information since he's the one that will give his opinion in the survey. Macro F1 scores obtained according to the input stream are reported in Table 2. As expected, the models trained using only the client's turns get better results than the ones that use the agent's turns, however combining agent and client turns shows a small improvement.

In the second experiment, we want to evaluate the robustness of the different models when we limit the vocabulary size 


\begin{tabular}{|l|c|c|c|c|c|c|}
\hline \multicolumn{7}{|c|}{ 3-label classification scheme } \\
\hline Model & Accuracy & SER & MacroF1 & F1 detractor & F1 passive & F1 promoter \\
\hline majority class & 42.7 & 30.9 & 19.9 & - & - & 59.9 \\
\hline SVM & 56.9 & $\mathbf{1 4 . 7}$ & $\mathbf{4 8 . 3}$ & 63.5 & $\mathbf{1 4 . 9}$ & 66.3 \\
\hline CNN & $\mathbf{5 7 . 5}$ & 15.5 & 46.2 & $\mathbf{6 4 . 4}$ & 7.2 & 67.0 \\
\hline RNN+att & $\mathbf{5 7 . 5}$ & 15.8 & 44.5 & 64.3 & 1.8 & $\mathbf{6 7 . 3}$ \\
\hline \hline \multicolumn{7}{|c|}{ 2-label+reject classification scheme } \\
\hline Model & Accuracy & SER & MacroF1 & F1 detractor & F1 passive & F1 promoter \\
\hline SVM 2x2 labels & 52.7 & $\mathbf{6 . 2}$ & 52.7 & 58.4 & $\mathbf{3 9 . 6}$ & 60.0 \\
\hline CNN 2x2 labels & $\mathbf{5 5 . 2}$ & 7.7 & $\mathbf{5 3 . 8}$ & $\mathbf{6 1 . 0}$ & 36.1 & 64.2 \\
\hline CNN 2 labels multitask & 55.0 & 7.6 & 53.5 & 59.8 & 36.3 & $\mathbf{6 4 . 4}$ \\
\hline RNN+att 2x2 labels & 53.5 & 6.5 & 53.0 & 58.2 & 39.4 & 61.3 \\
\hline RNN+att 2 labels multitask & 53.5 & 6.5 & 52.7 & 58.0 & 37.7 & 62.4 \\
\hline
\end{tabular}

Table 1. Models comparison with a 3-label and a 2-label+rejection classification schemes

\begin{tabular}{|l|c|c|c|}
\hline input/macroF1 & SVM & CNN & RNN+att \\
\hline all turns & 52.7 & 53.5 & 52.7 \\
\hline client turns & 51.1 & 52.3 & 52.5 \\
\hline agent turns & 47.2 & 48.2 & 47.6 \\
\hline
\end{tabular}

Table 2. Models comparison with macroF1 according to the input stream: all turns, only client turns, only agent turns

to the most frequent words. This robustness is an interesting feature as it is closely linked to the adaptation capabilities of the models to process new data from other domains. Indeed by using only frequent words, the models are less likely to be driven by topical words of a given domain. In this experiment we define several lexicons lexl to lex6 with several vocabulary sizes. For an experiment with lexicon $l e x_{i}$, all words that do not occur in $l e x_{i}$ are removed from the corpus prior to model training. Results from these experiments can be found in Figure 1. all corresponds to the whole corpus vocabulary (119165 words); lexl contains words occurring at least $10 \mathrm{~K}$ times (308 words); lex2, words occurring at least $20 \mathrm{~K}$ times (173 words); lex3, words occurring at least $30 \mathrm{~K}$ times (128 words); lex4, words occurring at least 40K times (108 words); lex 5 , words occurring at least $50 \mathrm{~K}$ times (92 words) and lex6, words occurring at least $100 \mathrm{~K}$ times (42 words).

As expected, the SVM which uses a simple bag of words as its input sees its macro F1 decrease when the size of the lexicon decreases. However, neural networks methods are much more stable. Both $\mathrm{CNN}$ and RNN show very few variations in their macro-F1 scores, except on the smallest lexicon where it loses 2 points compared to the previous lexicon with the CNN and 1 point with the RNN. These results probably mean that the neural networks are capable of using more than just the presence of some words to do their predictions on this task, capturing some of the structure of the conversations.

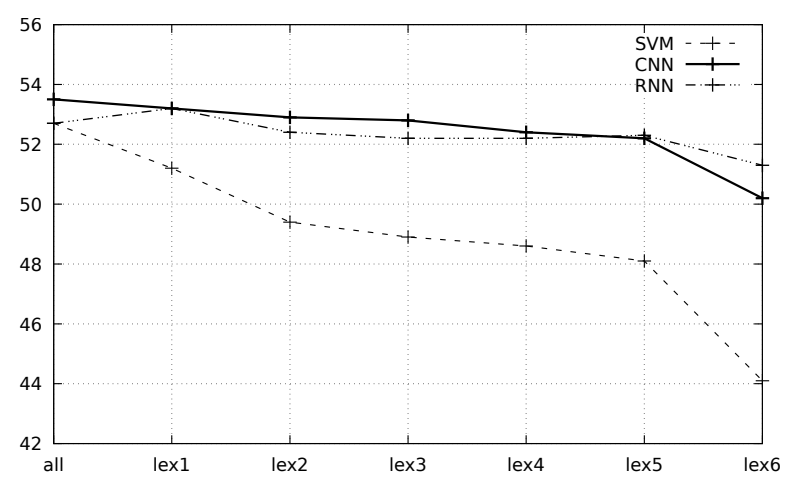

Fig. 1. Macro-F1 performance for SVM, CNN and RNN according to the lexicon size

\section{CONCLUSION}

We have shown in this study that self-reported satisfaction surveys can be used in a supervised classification paradigm for evaluating customer satisfaction directly from system logs. We have compared different classification schemes in order to classify each customer as detractors, passives and promoters and shown that a 2-label scheme manages to reduce by $50 \%$ the amount of serious errors compared to a standard 3-label classification model (from $14.7 \%$ to $6.2 \%$ error rate). Contrastive experiments have shown that CNN and RNN methods were more robust than SVM to the reduction of the input vocabulary, leading to a better generalization capability. Despite these good results, the general accuracy and macroF1 performance of the best models remains rather limited, indicating that there is still a lot of work to do in order to fully exploit the linguistic and interactive content of human-human conversations.

Acknowledgments This work has been partially funded by the Agence Nationale pour la Recherche (ANR) through the following programs: ANR-15-CE23-0003 (DATCHA), ANR-16-CONV-0002 (ILCB) and ANR-11-IDEX-0001-02 (A*MIDEX). 


\section{REFERENCES}

[1] Joseph Bockhorst, Shi Yu, Luisa Polania, and Glenn Fung, "Predicting self-reported customer satisfaction of interactions with a corporate call center," in $\mathrm{Ma}$ chine Learning and Knowledge Discovery in Databases, Yasemin Altun, Kamalika Das, Taneli Mielikäinen, Donato Malerba, Jerzy Stefanowski, Jesse Read, Marinka Žitnik, Michelangelo Ceci, and Sašo Džeroski, Eds., Cham, 2017, pp. 179-190, Springer International Publishing.

[2] Jordi Luque, Carlos Segura, Ariadna Sánchez, Martı Umbert, and Luis Angel Galindo, "The role of linguistic and prosodic cues on the prediction of self-reported satisfaction in contact centre phone calls," Proc. Interspeech 2017, pp. 2346-2350, 2017.

[3] Atsushi Ando, Ryo Masumura, Hosana Kamiyama, Satoshi Kobashikawa, and Yushi Aono, "Hierarchical lstms with joint learning for estimating customer satisfaction from contact center calls," Proc. Interspeech 2017, pp. 1716-1720, 2017.

[4] Shammur Absar Chowdhury, Evgeny A Stepanov, and Giuseppe Riccardi, "Predicting user satisfaction from turn-taking in spoken conversations.," in INTERSPEECH, 2016, pp. 2910-2914.

[5] Shourya Roy, Ragunathan Mariappan, Sandipan Dandapat, Saurabh Srivastava, Sainyam Galhotra, and Balaji Peddamuthu, "Qa rt: A system for real-time holistic quality assurance for contact center dialogues," in Thirtieth AAAI Conference on Artificial Intelligence, 2016.

[6] Géraldine Damnati, Aleksandra Guerraz, and Delphine Charlet, "Web chat conversations from contact centers: a descriptive study.," in LREC, 2016.

[7] Yoon Kim, "Convolutional neural networks for sentence classification," in Proceedings of the 2014 Conference on Empirical Methods in Natural Language Processing (EMNLP), 2014, pp. 1746-1751.

[8] Dzmitry Bahdanau, Kyunghyun Cho, and Yoshua Bengio, "Neural machine translation by jointly learning to align and translate," arXiv preprint arXiv:1409.0473, 2014.

[9] Kelvin Xu, Jimmy Ba, Ryan Kiros, Kyunghyun Cho, Aaron Courville, Ruslan Salakhudinov, Rich Zemel, and Yoshua Bengio, "Show, attend and tell: Neural image caption generation with visual attention," in International Conference on Machine Learning, 2015, pp. 2048-2057. 